\title{
Editorial
}

\section{Ethics committees and the treatment of congenital heart disease}

It is no secret among doctors that many established medical and surgical treatments have been inadequately evaluated. Even when they have been studied in population samples there is always an element of empiricism when they are tried in a particular case. It is often appropriate to share some of this uncertainty with our patients but hardly anyone would advocate providing an elaborate account of all the uncertainties and imponderables associated with everyday treatments. Most patients do not wish to receive such detailed information and generally trust the judgement of the profession.

The introduction of a new treatment is quite another matter. Most patients expect and have a right to know whether the treatment that they are being offered is novel; and the public is not happy to let doctors experiment with new treatments without a proper level of information and appropriate safeguards. The difficulty in defining what is novel as opposed to what is established and conventional applies equally to surgical and medical treatments. Occasionally treatments break entirely new ground but most evolve from pre-existing treatments and are steps in a continuing process of evolution. It is not possible to give unambiguous guidance on when a treatment is sufficiently novel to demand full patient information and consent, but perhaps a useful guideline might be to classify as novel any development that because of its novelty is regarded as worthy of publication in a journal. When there is doubt about whether or not a treatment is novel, in the interest of maintaining public confidence in medical research, it is preferable to err on the side of providing rather than withholding information. Most commentators would consider that the use of a novel arterial device to occlude aorto-pulmonary connections in children, as described in this issue, falls well within the category of innovation requiring a level of patient information and consent above that required for a conventional therapy.

Where then do ethics committees come into the argument? Surely clinicians can be relied upon to provide the appropriate level of information to allow informed consent for novel treatments. Some can and some cannot. Even when an innovation has been construed as research that requires informed consent, the information sheets provided for potential subjects are commonly under-informative and generally written in complex and technical language. Admittedly, the written information sheet is usually not the only communication received by the patient but it is the only evidence of communication and it would be surprising if there was not some correlation between poor written information and poor oral information. It is far better for all concerned if the introduction of a novel treatment is accompanied by an application to the local research ethics committee. This has the following benefits:

- The patient's interests are better protected. Firstly, a good level of patient information is more likely, particularly as a result of the contribution from lay members of the committee who will try to see things from the patient's perspective. Secondly, an independent opinion on the acceptability of risks involved is obtained, and although members of the committee do not generally have the specialist expertise of the applicant-that is sometimes an advantage.

- Ethics committee approval protects the interests of the clinician. Firstly, through an improved level of information to patients there is less likelihood of patient dissatisfaction. Secondly, the ethics committee may point out risks that can be reduced or avoided. Thirdly, ethics committee approval places any defence against criticism on a more secure footing.

- The independent evaluation of research and development proposals by an ethics committee enhances public confidence in research and without public confidence little progress can be made. Incidentally, it may also provide constructive, critical feedback to the researcher borne of the experience of the committee.

- Respected scientific journals do not publish reports of innovations that should have had ethics committee approval. Sadly, too often publication appears to be the prime motivation for application to the ethics committee, but as a means to an end this is no bad thing. In this issue of Heart the editors focus on the question of ethical approval for innovative treatments in paediatric cardiology and have deliberately chosen to publish illustrative material.

Applying to the local research ethics committee should not be seen as an obstacle or a threat to research. Most ethics committees are strongly in favour of potentially useful and well conducted research and seek only to ensure that the interests of participants are protected by a proper consideration of the risks and by the provision of adequate, intelligible, and honest information to patients. If these conditions are met and if the need to apply to the ethics committee is considered sufficiently early in the development of the project, applying to the ethics committee is not a barrier to progress.

Multicentre projects are more of a problem. The lot of a group of innovative clinicians attempting a multicentre study requiring approval from numerous ethics committees is not a happy one. Even if all committees were straightforward in their approach and were efficient, a considerable amount of work would be involved. If some of the committees are excessively fussy and long-winded it can be a nightmare. Fortunately, there are now advanced proposals for a degree of centralisation and streamlining of the process of ethical approval for multicentre projects and so life should soon become easier.

In the treatment of congenital heart disease, whether it be by drugs, open surgery, or a transvascular procedure, the need for ethics committee approval is as clear as anywhere else in medicine. Judging whether the proposed treatment is sufficiently new to require submission to an ethics committee will remain a problem, but there can be no complaint if clinicians err on the side of applying to the ethics committee when in doubt. Since paediatric cardiology is, happily, a clinical specialty in which improvements in outcome through research and development continue to occur at a rapid pace, ethics committees can anticipate a 
continuing challenge to their judgement for many years to come.

One point worthy of emphasis is that there is no difference between a novel surgical procedure and a novel drug treatment as far as the need to apply to the ethics committee is concerned. In either case, if significant new ground is being broken and the results are potentially publishable there is a need for informed consent from the patient and this should be underwritten by ethics committee approval. Surgeons may long have viewed themselves as creative artists working free of constraint, and clearly they must, like all practitioners, be allowed a considerable degree of clinical freedom. It is difficult to construct a logical argument that applies different standards to surgical and medical innovative procedures, however.

The principles of obtaining informed consent for the evaluation of novel treatments involving children are quite clear. Firstly, when the child is judged too young or too sick to be personally involved in giving consent, informed consent from the parents or legal guardians is acceptable. Secondly, when the child is judged to be old enough and well enough to contribute to the decision making process, albeit in a limited way, an attempt should be made to engage the child's opinion appropriately. Thirdly, every effort should be made to minimise the adverse effects of procedures undertaken solely for research purposes. Though an adult may be approached to submit to serial venepuncture for research purposes a child should generally not be expected to behave so altruistically. Everything possible should be done to minimise pain and discomfort.

To summarise, those contemplating the introduction of a novel treatment of any kind in paediatric cardiology should always consider whether or not ethics committee approval is required: when in doubt they should seek approval. They should ensure that their application to the ethics committee can be clearly understood by colleagues in other specialties and by lay people. In particular, they should ensure that the written information sheet for parents/patients provides a clear account of what is proposed. It must be honest about any important risks and written in plain English at a level that can be understood by a 12 year old.

P R F DEAR

Chairman of the Clinical Research Ethics Committee

St fames's University Hospital,

Beckett Street,

Leeds LS9 $7 T F$

\section{Leonardo da Vinci (1452-1519)}

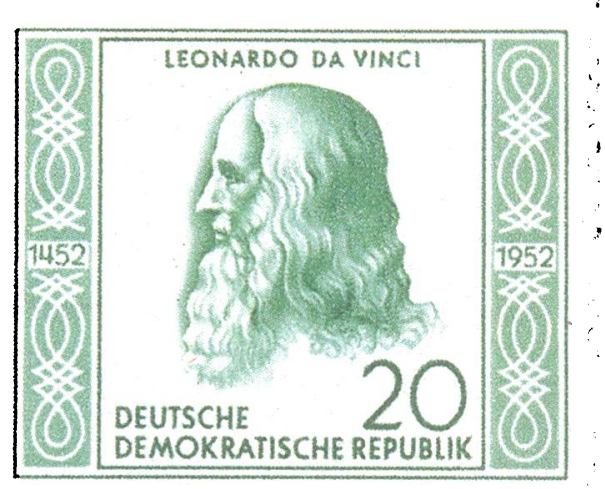

(A)

Cultural anniversaries were the theme of an East Germany set of four stamps issued in 1952. The 20 pfennig green stamp (A) marked the 500th anniversary of the birth of da Vinci.

In 1982 San Marino issued a Pioneers of Science set of 16 stamps, the second highest value of which featured Leonardo da Vinci (B). Other values included portraits of Hippocrates, Antoine Lavoisier, Marie Curie, Robert Koch, and Alexander Fleming.

Leonardo da Vinci is best known as a wonderful artist but his experiments, anatomical drawings, and notes (often in mirror writing) provide early descriptions of the structure and function of the heart and circulation. $\mathrm{He}$ termed the ventricles "the lower ventricles", the other chambers "the upper ventricles", the atrial appendages "the ears" and the heart as ". . . a muscle of pre-eminent power over other muscles". He was perhaps the first to describe a

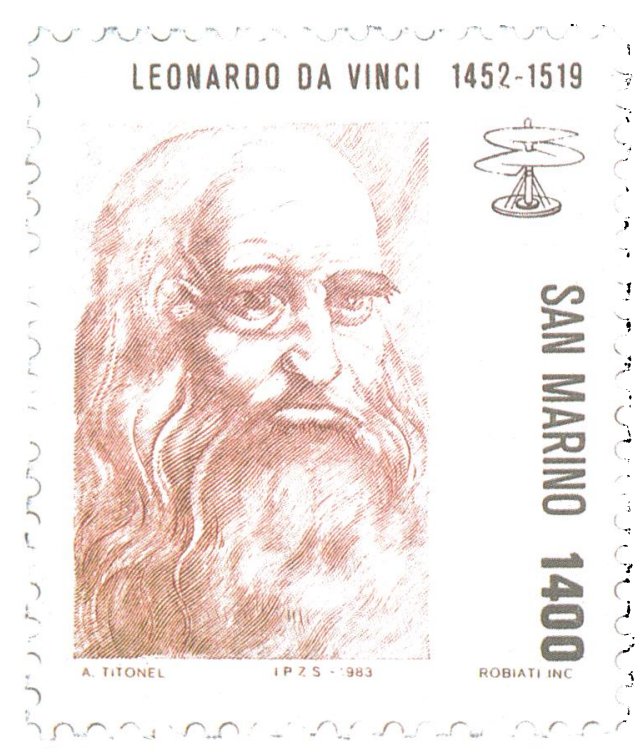

(B)

congenital heart lesion. His drawing in Quaderni d'Anatomia (1513) depicts a perforating channel between the atria, representing a patent foramen ovale. His experiments with animal hearts, in which he allowed fragments of leaves in water to circulate through the heart, demonstrated that blood flow was unidirectional and defined cardiac valve function. His interest in anatomy was inspired by the anatomist Marcoantonio della Torre (14731511) who was professor of anatomy in Pisa and then Padua and who commissioned da Vinci to provide the illustrations for his text on anatomy based on dissection. 